\title{
Neural-Specific Carbohydrate Moiety Shared by Many Surface Glycoproteins in Drosophila and Grasshopper Embryos
}

\author{
Peter M. Snow, Nipam H. Patel, Allan L. Harrelson, and Corey S. Goodman \\ Department of Biological Sciences, Stanford University, Stanford, Californa 94305
}

\begin{abstract}
Antiserum against horseradish peroxidase (anti-HRP Ab) labels the surfaces of neurons in both Drosophila and grasshopper (Jan and Jan, 1982). Here we show that the anti-HRP Ab (1) immunoprecipitates at least 17 different membrane glycoproteins from the Drosophila embryo CNS (and a similar array from grasshopper), and (2) recognizes a neural-specific carbohydrate moiety expressed by most if not all of these proteins. Although the anti-HRP $\mathbf{A b}$ stains all axon pathways, 2 of the anti-HRP glycoproteins, fasciclin I and II, are expressed on specific subsets of axon pathways in the grasshopper embryo (Bastiani et al., 1987).
\end{abstract}

Antiserum against horseradish peroxidase (anti-HRP Ab) labels the surface of all axon pathways in the central and peripheral nervous system of the Drosophila and grasshopper embryos (Jan and Jan, 1982); it is a remarkable neural-specific probe that stains the entire surface of individual embryonic neurons including their axons, growth cones, and filopodia. Thus this antibody has been widely used as a powerful marker in studies on neuronal development in insects (e.g., Bentley and Keshishian, 1982; Blair and Palka, 1985a, b; Caudy and Bentley, 1986a, b).

Given the neural specificity of the anti-HRP Ab, we wondered about the biochemical nature of the epitope it recognizes. Does the antibody recognize an antigen expressed by only a single surface glycoprotein, or does it recognize a common antigen found on many surface glycoproteins? To answer these questions, we used a variety of biochemical and immunocytochemical methods to characterize the anti-HRP epitope.

In this paper we show that the anti-HRP $\mathrm{Ab}$ recognizes a neural-specific carbohydrate moiety in both grasshopper and Drosophila. This carbohydrate is expressed by most if not all of the 17 glycoproteins immunoprecipitated by the anti-HRP Ab. Although the anti-HRP Ab stains all axon pathways, at least 2 of the anti-HRP glycoproteins, fasciclin I and II, are expressed with greater specificity. Fasciclin I and II are expressed on specific subsets of axon pathways in the grasshopper embryo (Bastiani et al., 1987). However, not all fasciclin I and II molecules have this carbohydrate, particularly when they are expressed on non-neural cells.

\footnotetext{
Received Feb. 24, 1987; revised May 18, 1987; accepted June 11, 1987.

We thank Denise Johnson for help in generating the 5B2 MAb, Michael Bastiani for help in purifying fasciclin I and II, David Smouse and John Thomas for help with the initial TM3 experiments, and Violette Paragas and Zaida Traquina for technical assistance. P.M.S. and A.L.H. are ACS Postdoctoral Fellows and N.H.P. is a NSF Predoctoral Fellow. Supported by grants from the NIH, the McKnight Foundation, and the March-of-Dimes Birth Defects Foundation to C.S.G.

Correspondence should be addressed to Corey S. Goodman at the above address. Copyright (C) 1987 Society for Neuroscience $0270-6474 / 87 / 124137-08 \$ 02.00 / 0$
}

\section{Materials and Methods}

Immunofluorescence. Embryos were prepared for immunofluorescence as whole-mounts and as dissections with minor modifications of previously published methods (Mitchison and Sedat, 1983; Goodman et al., 1984). Embryos were washed in PBS (pH 7.4), 0.2\% BSA, $0.1 \%$ Triton X-100 (called PBT) for $1 \mathrm{hr}$, preincubated in PBT containing $5 \%$ normal goat serum (NGS) for 30 min, incubated in primary antibody (for whole-mounts, 1:500 fluorescein isothiocynate (FITC)-conjugated goat anti-HRP, $25^{\circ} \mathrm{C}, 2 \mathrm{hr}$; for dissections, 1:1 5B2 MAb supernatant, $4^{\circ} \mathrm{C}, 16 \mathrm{hr}$ ) (anti-HRP, Cappel), and washed in PBT for $2 \mathrm{hr}$. Wholemounts were viewed at this time, whereas dissections were now prcincubated in PBT plus NGS for $30 \mathrm{~min}$, incubated in secondary antibody (1:200 FITC-conjugated goat anti-mouse Ig plus 1:500 rhodamine-conjugated goat anti-HRP prepared from unlabeled anti-HRP) (anti-HRP Cappel) at $25^{\circ} \mathrm{C}$ for $2 \mathrm{hr}$, and washed in PBT for $2 \mathrm{hr}$.

Grasshopper embryos were dissected and fixed in $2 \%$ paraformaldehyde for 15-30 min, then rinsed in saline. Embryos were rinsed briefly with a solution of $1 \% \mathrm{BSA}, 0.4 \%$ saponin, $5 \%$ normal goat serum in PBS. Then a 1:400 (rat anti-fasciclin I) or 1:800 (rat anti-fasciclin II) dilution of antiserum was applied in this same BSA/saponin/NGS solution and incubated overnight at $4^{\circ} \mathrm{C}$. After rinsing in BSA/saponin/ NGS solution for $2 \mathrm{hr}$, a 1:400 dilution of FITC-conjugated goat antirat Ig secondary antibody (Cappel) in BSA/saponin/NGS solution was added for $1 \mathrm{hr}$ at $33^{\circ} \mathrm{C}$, followed by a final rinse for $2 \mathrm{hr}$.

Carbohydrate isolation. HRP, $250 \mathrm{mg}$ (Sigma), was digested with 2.5 mg pronase (type XIV; Sigma) for $96 \mathrm{hr}$, with additions of $2.5 \mathrm{mg}$ pronase at $24 \mathrm{hr}$ intervals. The digest was then further treated by the addition of $0.1 \% \mathrm{SDS}$ at $65^{\circ} \mathrm{C}$ for $10 \mathrm{~min}$, followed by the addition of $2.5 \mathrm{mg}$ of proteinase $\mathrm{K}$ (Sigma) and further incubation for $24 \mathrm{hr}$ at $51^{\circ} \mathrm{C}$. The digest was lyophilized and the glycopeptides precipitated in $90 \%$ ethanol. Further purification was performed using a $2.5 \times 50 \mathrm{~cm}$ Sephadex G-25 fine (Pharmacia) sizing column. The carbohydrate-containing fractions were identified using the phenol-sulfuric acid method (Ashwell, 1966). These fractions were pooled, lyophilized, resuspended in water $(1 \mathrm{ml})$, and an aliquot corresponding to $25 \mu \mathrm{g}$ of starting material was analyzed on a $20 \%$ polyacrylamide gel run according to Laemmli (1970) and visualized by silver-staining. This gel showed that no residual polypeptides were present (a $20 \%$ gel would have allowed us to resolve peptides of 10 amino acids or less).

For carbohydrate competition experiments, the anti-HRP Ab was prepared at the same final dilution in $250 \mu \mathrm{l}$ with the addition of carbohydrates isolated from $1.5 \mathrm{mg}$ of initial starting HRP. For control embryos, anti-HRP Ab was competed with carbohydrates isolated from ovalbumin (equal starting amounts compared to HRP). For periodate treatment, embryos were washed in $20 \mathrm{~mm}$ sodium acetate buffer $(\mathrm{pH}$ 4.5) containing $0.1 \mathrm{M} \mathrm{NaCl}$ at $4^{\circ} \mathrm{C}$ for $30 \mathrm{~min}$. Sodium meta-periodate was added to $10 \mathrm{~mm}$ for $30 \mathrm{~min}$. Control embryos remained in the buffer throughout. Both were washed in PBS and then stained as above.

Biochemical techniques. Drosophila $10-13 \mathrm{hr}$ embryo central nervous systems (nerve cords) were enriched as previously described (Goodman et al., 1984). All steps were performed at $0-4^{\circ} \mathrm{C}$. The purified tissue was resuspended in $10 \mathrm{~mm}$ triethanolamine (TEA) containing $1 \mathrm{~mm}$ phenymethylsulfonyl chloride (PMSF) and $1 \mu \mathrm{g} / \mathrm{ml}$ of the following protease inhibitors: antipain, chymostatin, leupeptin, pepstatin, TLCK, and TPCK. The nerve cords were homogenized and centrifuged for $15 \mathrm{~min}$ at $1500 \times \mathrm{g}$. The supcrnatant was reserved and the pellet was rehomogenized and again centrifuged. The supernatants were combined and 
centrifuged at $100,000 \times g$ for $1 \mathrm{hr}$. The membrane pellet was resuspended in PBS containing $1 \mathrm{mM}$ PMSF for iodination. Iodination of 1 $\mathrm{mg}$ of membrane protein with lactoperoxidase in $300 \mu \mathrm{l} \mathrm{PBS} / \mathrm{PMSF}$ was performed essentially as described (Haustein et al., 1975). After iodination, the membranes were collected by centrifugation at $12,000 \times g$ for $15 \mathrm{~min}$ and the membrane proteins were solubilized in $0.5 \mathrm{ml}$ of $10 \mathrm{~mm}$ TEA, $0.15 \mathrm{M} \mathrm{NaCl}, 2 \% \mathrm{NP}-40,0.5 \%$ deoxycholate (DOC), pH 8.2, containing the protease inhibitors described above. After $1 \mathrm{hr}$ on ice, the lysate was subjected to centrifugation for $30 \mathrm{~min}$ at $100,000 \times g$.

Immunoprecipitations were performed using preformed antibody complexes as described (van Agthoven et al., 1981). The immunoprecipitated proteins were analyzed by SDS-PAGE according to a modification of the method of Laemmli (1970). Periodate treatments were performed on membranes that had been resuspended by homogenization in $20 \mathrm{~mm}$ sodium acetate buffer, $\mathrm{pH} 4.5$, containing $0.1 \mathrm{M} \mathrm{NaCl}$. Sodium meta-periodate was added to $10 \mathrm{~mm}$ and the reaction was allowed to proceed in the dark for $30 \mathrm{~min}$ at $4^{\circ} \mathrm{C}$. The membranes were collected and iodinated as described above. Control membranes were incubated in the sodium acetate buffer without the addition of metaperiodate.

Fasciclin I and II were purified from 40-50\% grasshopper embryos by affinity chromatography using the $3 \mathrm{~B} 11$ and $8 \mathrm{C} 6 \mathrm{MAbs}$, and used to generate a rat antiserum as described by Bastiani et al. (1987). Purified protein was precipitated with $20 \%$ trichloroacetic acid for $30 \mathrm{~min}$ at $4^{\circ} \mathrm{C}$ and collected by centrifugation at $13,000 \times g$ for $15 \mathrm{~min}$. The pellet was washed 3 times in ice-cold acetone and lyophilized.

Labeling with ${ }^{125} \mathrm{I}$ and chloramine $\mathrm{T}$ was performed as described (Greenwood et al., 1963). Sperm whale myoglobin (Beckman Instruments), $50 \mu \mathrm{g}$, was added as carrier to each aliquot prior to immunoprecipitation to prevent nonspecific adsorption. Immunoprecipitation and SDS-PAGE were performed as described above. Autoradiography was performed at $-80^{\circ} \mathrm{C}$ using Kodak XAR-5 film in combination with intensifier screens (Cronex Lightning Plus; Dupont Chemical Co.).

Two-dimensional gel electrophoresis was performed essentially according to O'Farrell (1975), with a pH gradient from $\mathrm{pH} 4$ to 9 , formed by a mixture of $\mathrm{pH} \mathrm{4-6.5}$ and $\mathrm{pH}$ 6.5-9 ampholytes (Pharmacia) in a ratio of $1: 1(\mathrm{vol} / \mathrm{vol})$. Isoelectric focusing was performed at $300 \mathrm{~V}$ (constant voltage) for $16 \mathrm{hr}$, followed by $800 \mathrm{~V}$ for $4 \mathrm{hr}$.

\section{Results}

Anti-HRP Ab immunoprecipitates many different neural surface glycoproteins

The anti-HRP Ab stains the surface of all axon pathways in the CNS and PNS of the Drosophila and grasshopper embryos (Jan and Jan, 1982), and thus reveals the segmental ladderlike arrangement of longitudinal and commissural axon fascicles in the CNS (Figs. $1, A, B ; 2 A$ ); in the Drosophila embryo, it also stains the Garland gland cells (Fig. 1, $A, B$ ).

In numerous hybridoma fusions using membranes of massisolated Drosophila embryonic CNSs as immunogens, we generated several MAbs (e.g., the 5B2 and 3B2 MAbs) whose staining patterns were identical to one another (Fig. 1F) and closely resembled that of anti-HRP (Fig. $1 E$ ).

In order to determine the relationship between the molecules recognized by these $\mathrm{Abs}$, membranes were prepared from massisolated embryonic CNSs, iodinated, and solubilized in NP-40. Following immunoprecipitation with either anti-HRP, 5B2, or $3 \mathrm{~B} 2$, the specific proteins recognized by the Abs were analyzed by one-dimensional SDS-PAGE (Fig. $3 A$ ).

Such an analysis revealed that the anti-HRP Ab reproducibly immunoprecipitated many different proteins with molecular weights ranging from about 50 to $150 \mathrm{kDa}$ (Fig. $3 A$ ) from the Drosophila embryo CNS. Anti-HRP Ab also immunoprecipitated a similar array of proteins from the grasshopper CNS (data not shown). Similarly, the 5B2/3B2 MAbs immunoprecipitated many proteins from the Drosophila embryo CNS in the same molecular-weight range, although fewer than did anti-HRP; moreover, all of thesc bands comigratcd with a subset of the anti-HRP bands (Fig. $3 A$ ).

In order to better characterize and compare the anti-HRP and 5B2/3B2 proteins in Drosophila, two-dimensional (2-D) gel electrophoresis was used. On 2-D analysis, at least 17 different reproducible and specific anti-HRP proteins could be counted (arrows and arrowheads Fig. 4B), some of which were so faint that it was possible that further low-abundance proteins were being missed. Two-dimensional analysis of the $3 \mathrm{~B} 2$ proteins showed 11 reproducible proteins (arrowheads in Fig. $4 C$; the 5B2 MAb showed a similar pattern of proteins), all of which appeared identical to anti-HRP proteins, thus suggesting that the 5B2/3B2 MAbs immunoprecipitate a subset of the antiHRP proteins (open arrows, in Fig. $4 B$ show those proteins immunoprecipitated by anti-HRP but not by the $5 \mathrm{~B} 2 / 3 \mathrm{~B} 2$ MAbs).

Although all of the $5 \mathrm{~B} 2 / 3 \mathrm{~B} 2$ proteins were identical to the anti-HRP proteins, there were consistent differences in the relative quantities of particular proteins immunoprecipitated by the different Abs (compare Fig. 4, $B$ with $C$ ). In some cases, greater amounts of a particular protein were reproducibly precipitated by the 5B2/3B2 MAbs (asterisks, Fig. $4 C$ ), whereas for other proteins greater amounts were reproducible when precipitated by the anti-HRP Ab. Onc possiblc interpretation of this result is that these proteins are heterogeneous in their expression of these 2 epitopes.

\section{Anti-HRP Ab recognizes a carbohydrate moiety}

Most of the anti-HRP and 5B2/3B2 proteins ran as either discrete smears or linear arrays of dots of increasing charge (Fig. $4, B, C$ ), indicating that they might be glycosylated in heterogeneous forms. In order to examine the glycosylation of the HRP proteins, solubilized, iodinated membrane proteins were passed over a concanavalin A (Con A) column and the bound glycoproteins were eluted with $\alpha$-methyl mannoside. The eluted proteins were then subjected to immunoprecipitation with antiHRP antiserum and analyzed by $2-\mathrm{D}$ gel electrophoresis. This approach showed that most if not all of the proteins immunoprecipitated by anti-HRP were also bound by Con A, indicating their glycoprotein nature (data not shown; same as for Fig. 4B).

The next subject of interest was the determination of the epitope that is recognized by anti-HRP, that is, of what epitope is shared in common between a plant glycoprotein and a series of Drosophila neuronal surface glycoproteins. Three experimental approaches were used to show that the epitope included a carbohydrate: (1) competition experiments with isolated HRP glycopeptides versus isolated HRP-deglycosylated proteins, (2) binding studies of glycoproteins with oxidized carbohydrates, and (3) experiments using the TM 3 balancer chromosome.

In the first experimental approach, HRP was digested with pronase and proteinase $\mathrm{K}$ to completion (see Materials and Methods). Glycopeptides were then separated from free amino acids, and small oligopeptides by gel-exclusion chromatography. The absence of residual polypeptides was confirmed by analyzing this material on a $20 \%$ polyacrylamide gel run according to Laemmli (1970) and visualized by silver-staining. The purified glycopeptides were used to compete for binding to anti-HRP antibodies on immunohistological preparations (Fig. 1C), as well as in immunoprecipitations with anti-HRP antisera (data not shown). Because we were unable to quantify the amount of glycopeptide recovered, and thus the concentration required for 

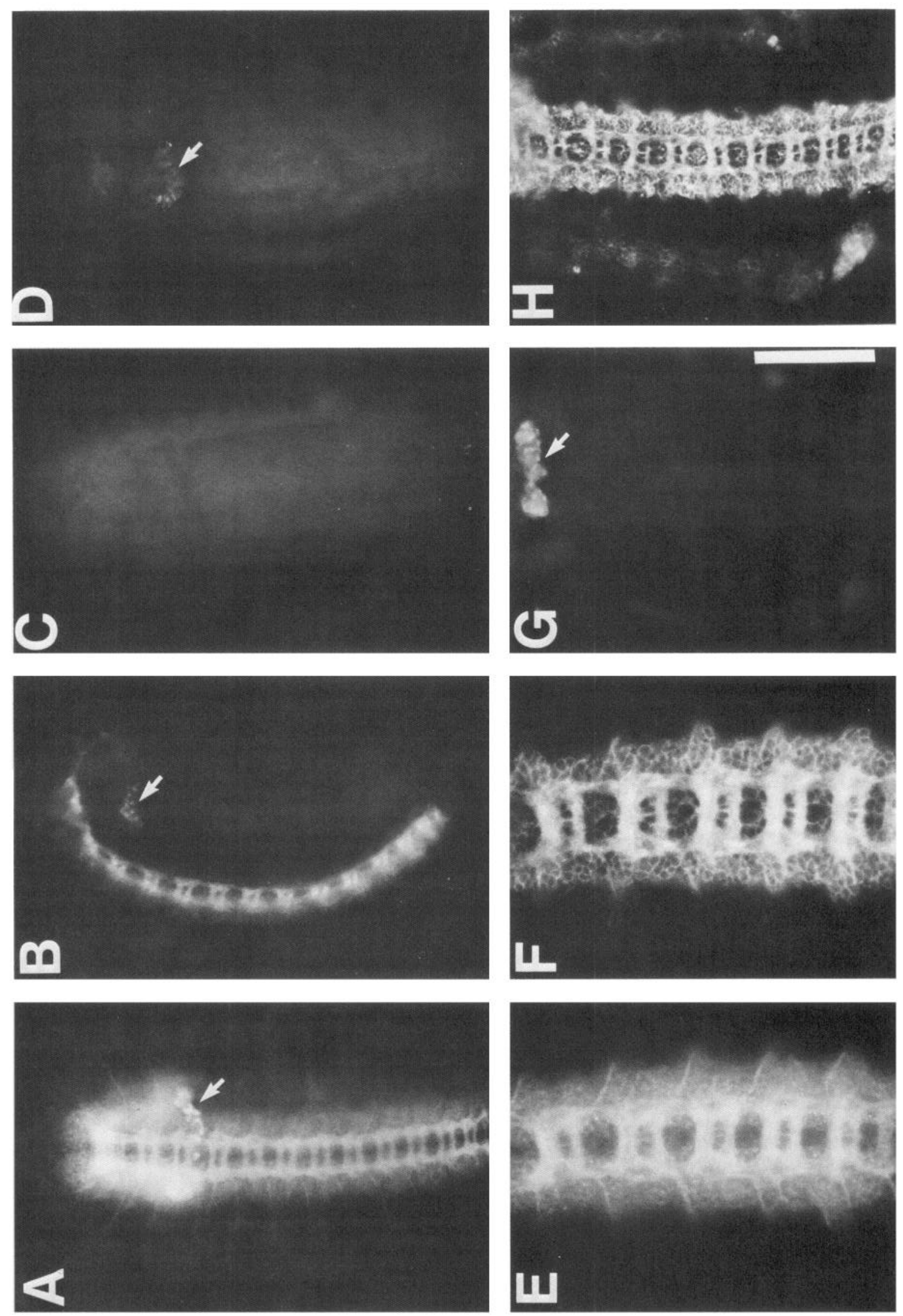

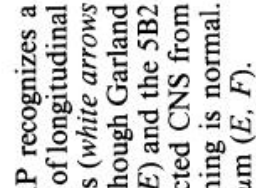

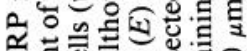

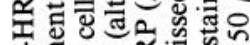
웅

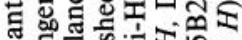
त कo on

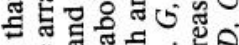
8 연. 군 0203

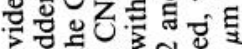

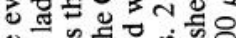

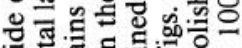
50 웅 05 它

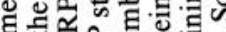

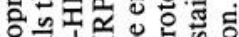

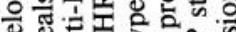

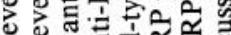

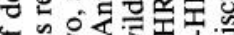

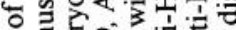
北 0 ह

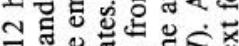

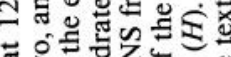
त

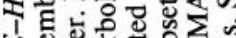
幽它氙过 $\Sigma$

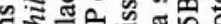

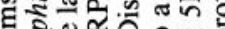

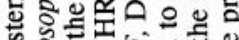

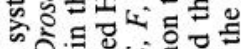
可计界 o읠 记

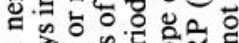
讨

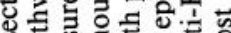

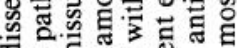
च

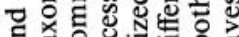

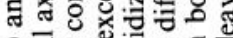

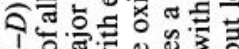
I 0 . u造 嵌

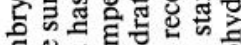
통 충 을

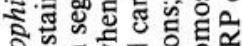
20 3 別

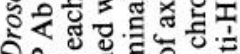
的需

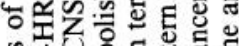
.

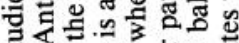
के 8 .

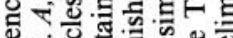

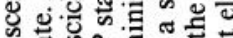

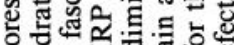

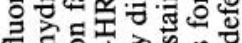

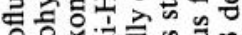
응 可 전

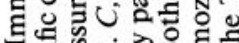
制. 说是言 웅

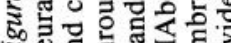

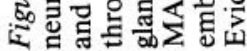



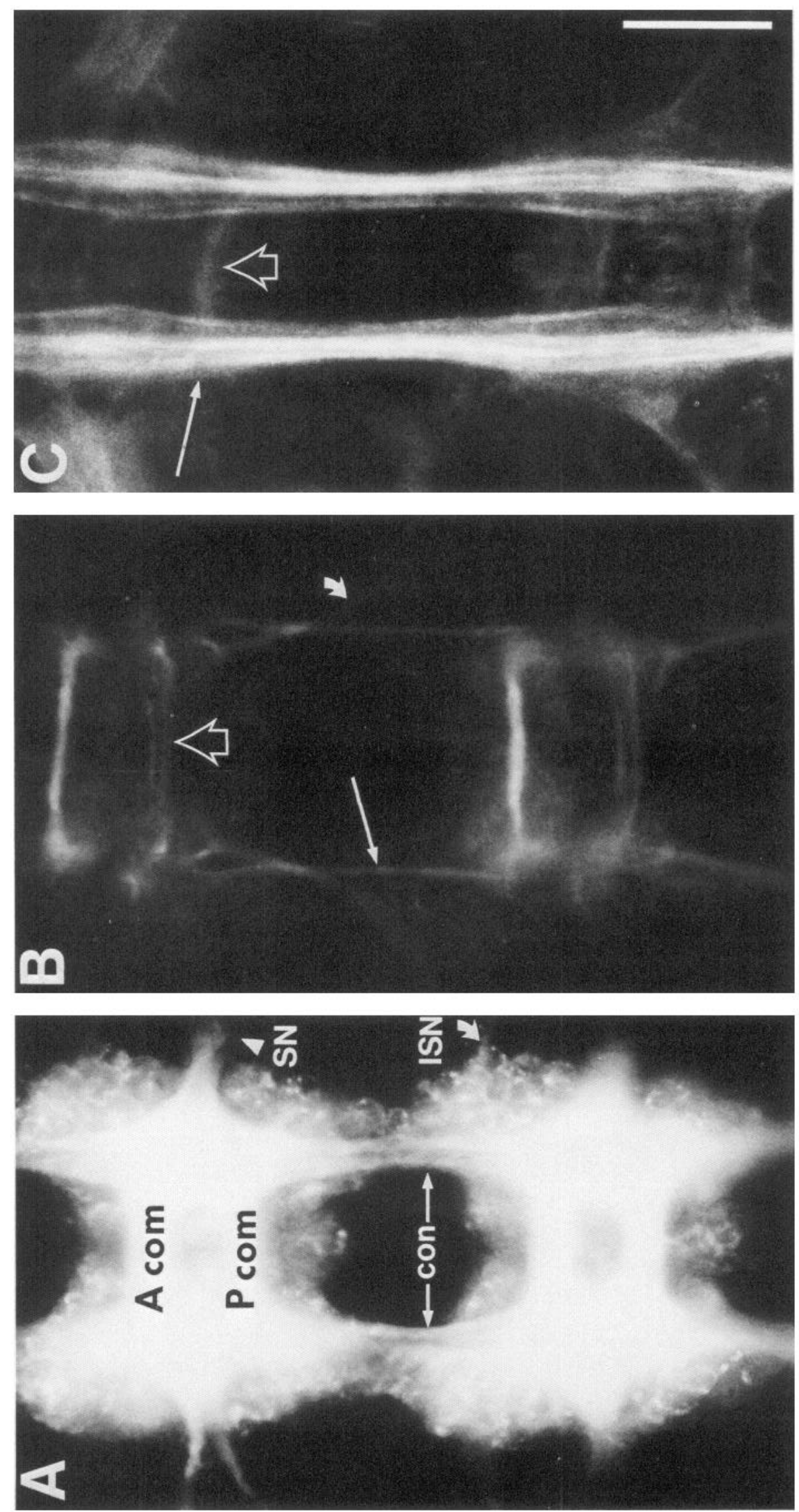

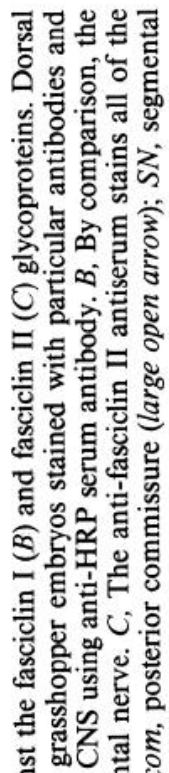
웅

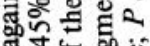
于

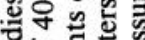
o녕. 藏䓵焉

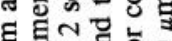

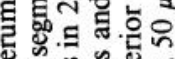

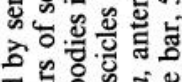

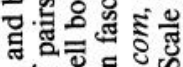
ति잉

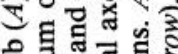
<.: 경 क्षे

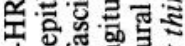
它 둴둥 政 त् बन 열

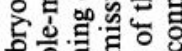

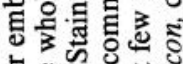

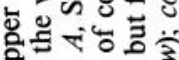

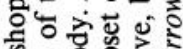
象实然 뜰

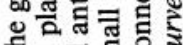
점 믕 웅 的 论 3. क क 2. 过

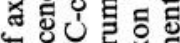
o 跑这的 苯的需 क

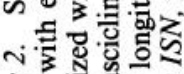
今 जु

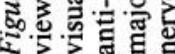




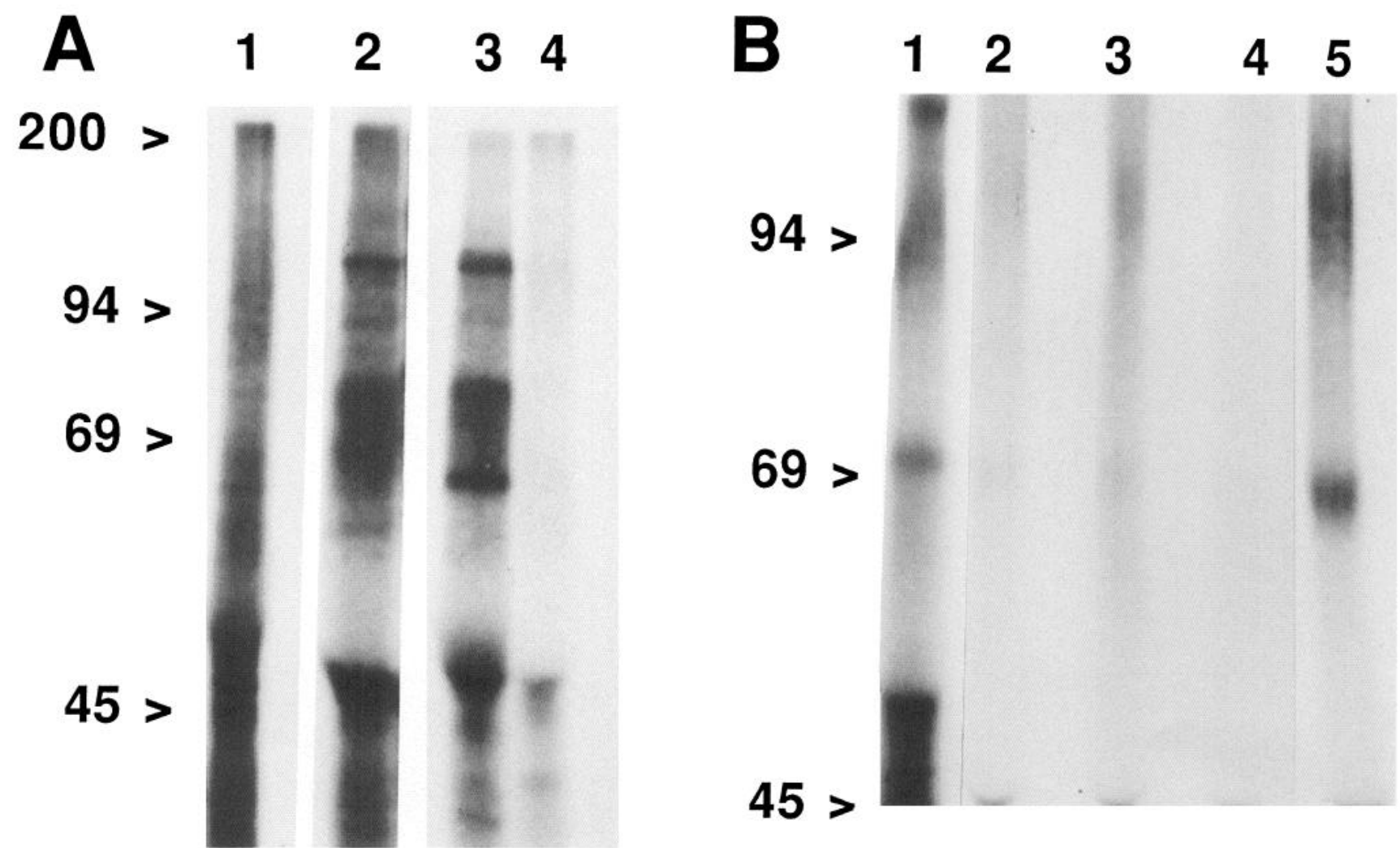

Figure 3. Analysis by immunoprecipitation of the proteins recognized by anti-HRP antiserum and the $5 \mathrm{~B} 2$ monoclonal antibody. $A$, Analysis by SDS-PAGE of the glycoproteins immunoprecipitated by anti-HRP antiserum and the 5B2 MAb. Nerve cord membranes were labeled with ${ }^{225}$ and the membrane proteins solubilized in a Nonidet P-40 (NP-40)-containing buffer. Immunoprecipitation was performed using a preformed antibody complex of either normal mouse serum (lane 1), 5B2 MAb (lane 2), or goat anti-HRP (Cappel) (lanes 3, 4). Immunoprecipitations with anti-HRP were also performed on iodinated membranes that were incubated either without (lane 3) or with (lane 4) sodium meta-periodate. Immunoprecipitates were analyzed under reducing conditions on a $7.5 \%$ polyacrylamide gel. $B$, The fasciclin I glycoprotein purified from the grasshopper embryo expresses the neural-specific carbohydrate recognized by anti-HRP Ab. Purified fasciclin I (70 kDa) was labeled with ${ }^{125}$ I by the chloramine-T method. Aliquots of the labeled protein were subjected to sequential immunoprecipitation with anti-HRP antiserum (lanes $1-$ 4). After depletion of material reactive with anti-HRP (e.g., lane 4,), the material was subjected to immunoprecipitation with antiserum generated against gel-purified fasciclin I (lane 5). In control experiments, labeled protein was treated with preformed complexes of normal mouse serum for an equal number of rounds of sequential immunoprecipitation, and subsequently immunoprecipitated with anti-HRP to control for nonspecific depletion of antigen. In these experiments, even after 4 rounds of precipitation, anti-HRP-reactive fasciclin I was still observed (not shown). Immunoprecipitates were analyzed under reducing conditions on $7.5 \%$ polyacrylamide gels. Lanes $1-4$ were exposed for $24 \mathrm{hr}$, while lane 5 was exposed for $4 \mathrm{hr}$. Molecular-weight markers are indicated in kilodaltons (kDa).

blockade, we used as a control the purified glycopepetides from the same starting amount of glycoprotein ovalbumin.

In a converse experiment, HRP was treated with trifluoromethanesulfonic acid (TFMS), which has been shown to cleave both $\mathrm{N}$ - and O-linked sugars (Edge et al., 1981). The deglycosylated protein was then used to compete for antibody binding on tissue. A control for protein degradation was an aliquot of the deglycosylated HRP seen to run as a single, discrete band of the appropriate molecular weight on a polyacrylamide gel.

The isolated HRP glycopeptides blocked both the biochemical and immunocytochemical assays (blocking staining of both the CNS and Garland cells in Fig. $1 C$; the ovalbumin glycopeptides had no effect), whereas the deglycosylated protein had no effect (data not shown). This experiment was repeated with the same results using the immunofluorescence assay with the grasshopper embryo (data not shown).

A second approach involved treatment of the glycoproteins with sodium meta-periodate, which oxidizes terminal reducing sugars (Spiro, 1966). Such a treatment has been shown, in some cases, to result in the destruction of the epitope recognized by antibodies directed against larger carbohydrate moieties (Kruse et al., 1984; Wiedenmann and Franke, 1985). Thus, nerve cord membranes or whole-mount embryos were given a mild periodate treatment and subsequently tested either for their ability to be labeled with anti-HRP (whole-mounts), or for the presence of the expected proteins upon immunoprecipitation (membranes).

Treatment with periodate blocked the immunoprecipitation of all of the anti-HRP glycoproteins from isolated CNS membranes (Fig. $3 A$, lane 4), and also blocked the tissue immunofluorescence of the nervous system with anti-HRP Ab (Fig. 1D). Periodate only partially reduced the staining of the Garland gland, suggesting that the epitope recognized by anti-HRP on these gland cells may be different from the epitope recognized on neuronal membranes. In contrast to the anti-HRP experiments, pertiodate treatment did not block the ability of the 5B2 MAb to immunoprecipitate its normal array of neuronal membrane glycoproteins (data not shown; same as Fig. 3A, lane 2). However, this negative result alone does not rule out the possibility that the $5 \mathrm{~B} 2$ epitope includes a carbohydrate; rather, it only suggests that it does not include terminal sugars. In this case, however, we were not able to purify sufficient quantities 
A

pH 4 9

200

116

94

69

45

C

pH 4

\section{9}

200

116

94

69
B

pH 4

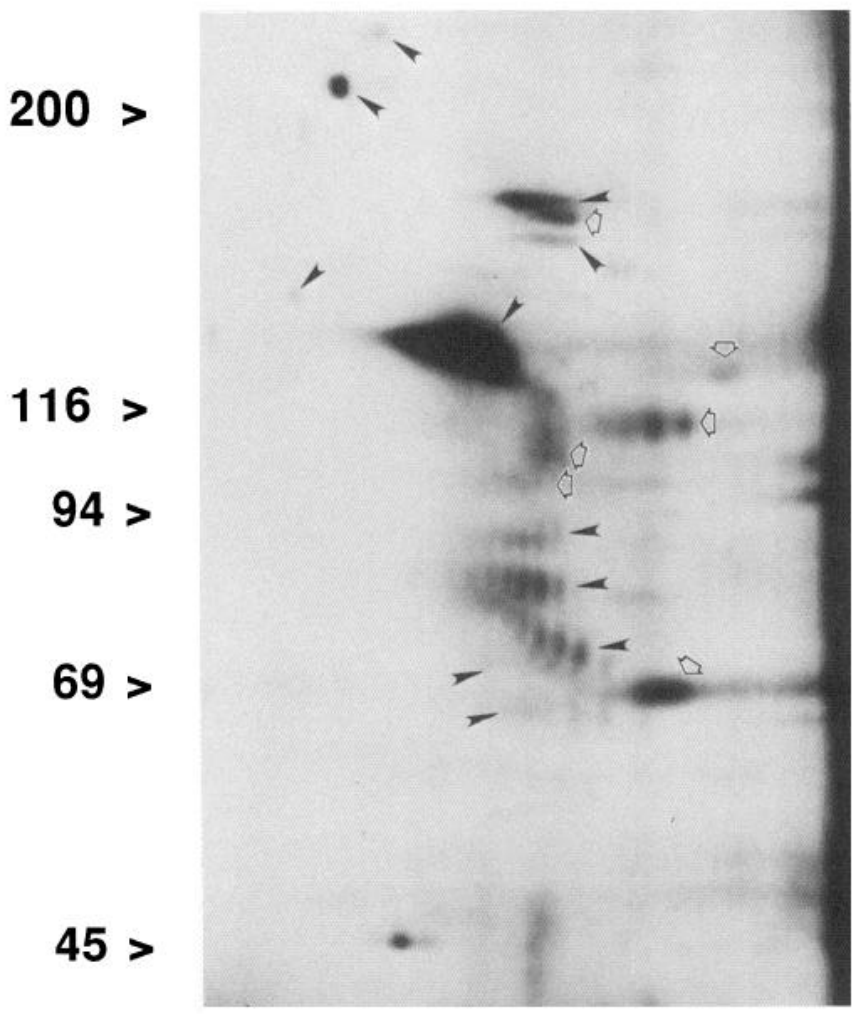

Figure 4. Analysis by 2-dimensional gel electrophoresis of the proteins recognized by anti-HRP and the 3B2 MAb. Immunoprecipitations from ${ }^{125}$ I-labeled nerve cord membranes were performed using $(A)$ normal mouse serum as control, $(B)$ anti-HRP, or $(C) 3 \mathrm{~B} 2 \mathrm{MAb}$. The immunoprecipitates were analyzed by $2-\mathrm{D}$ gel electrophoresis according to O'Farrell (1975), using a pH gradient of 4-9 in the first (isoelectric focusing) dimension. The second dimension was a $6 \%$ polyacrylamide gel. Molecular-weight markers are shown in kilodaltons (kDa). B, Arrows of both types mark the 17 glycoproteins reproducibly immunoprecipitated by anti-HRP Ab. Open arrows mark those proteins not immunoprecipitated by the 3B2 MAb. $C$, Arrowheads mark the 11 glycoproteins immunoprecipitated by the $3 \mathrm{~B} 2 \mathrm{MAb}$. In some cases, greater amounts of a particular protein were reproducibly precipitated by the 3B2 MAbs than by the anti-HRP Ab (marked by asterisks).

of the $5 \mathrm{~B} 2 / 3 \mathrm{~B} 2$ proteins to carry out the same types of proteinversus-carbohydrate competition experiments that we did with HRP.

These results suggest that the epitope recognized by the antiHRP Ab is a carbohydrate. Although it is formally possible that the anti-HRP Ab is recognizing different carbohydrate epitopes on different glycoproteins, we think it unlikely, since such a conclusion would imply that a plant glycoprotein and an array of Drosophila neural surface glycoproteins share more than one carbohydrate epitope with a terminal reducing sugar. At present the nature of the 5B2/3B2 epitope(s) is unknown.

A third approach to this question involved studying Drosophila embryos homozygous for the balancer chromosome TM3 (with multiple point mutations and break points). Such embryos lack all anti-HRP staining in their nervous system (Fig. 1G) (although the Garland gland cells continue to stain). The 5B2 $\mathrm{MAb}$, which recognizes a different epitope on a subset of the anti-HRP proteins, continues to stain these embryos normally (Fig. $1 H$ ), suggesting that many if not all of the anti-HRP pro- 
teins may be present in this mutant and that the defect may lic in the glycosylation. The most likely explanation for these observations is that the anti-HRP epitope includes a posttranslational modification of all of the anti-HRP glycoproteins. This modification must be genetically altered by the TM3 chromosome, since the epitope is deleted, but most, perhaps all of the proteins are present in these mutant embryos. These results further confirm the theory that the anti-HRP epitope includes a carbohydrate moiety common to many different neural surface glycoproteins.

\section{Fasciclin $I$ and $I I$ are among the glycoproteins recognized by the anti-HRP $A b$}

In previous studies on neuronal development in the grasshopper embryo, 2 MAbs, 3B11 and 8C6, were used to study the expression of and biochemically characterize 2 different cell surface glycoproteins, called fasciclin I and fasciclin II, of $M_{\mathrm{r}} 70$ and 95 $\mathrm{kDa}$, respectively (Bastiani et al., 1987). Fasciclin I and II are expressed on different subsets of axon fascicles during development in a spatiotemporal pattern that makes them good candidates for molecules involved in the events of selective fasciculation (e.g., Goodman et al., 1984).

That fasciclin I and II might be anti-HRP glycoproteins was suggested when immunoprecipitates using the anti-IIRP $A b$, 3B11 MAb, and 8C6 MAb were run in parallel on 1-D SDSPAGE; 2 of the grasshopper anti-HRP proteins comigrated with fasciclin I and II. Comparison of these species by 1-D peptide map analysis indicated that fasciclin I and the HRP protein have identical fragments upon limited proteolysis with Staphylococcus V8 protease (data not shown).

Affinity-purified fasciclin I and II proteins were used to prove that both of these proteins are in fact anti-HRP glycoproteins. The anti-HRP Ab immunoprecipitated both purified fasciclin I (Fig. 3B) and II (data not shown) glycoproteins. This is the same column and gel-purified protein that was used to generate the antisera against each protein; these antisera stain the same specific subsets of axon pathways, respectively, as do the original MAbs used to initially characterize the proteins (Fig. 2, $B, C$; Bastiani et al., 1987).

The demonstration that fasciclin I and II are anti-HRP proteins is very interesting, but also presents a paradox. Immunocytochemical studies had shown that, in addition to their expression on specific subsets of axon fascicles, both fasciclin I and II are expressed outside of the developing nervous system on the surface of non-neural ectodermal cells (Bastiani et al., 1987). In contrast, the anti-HRP Ab typically does not stain these non-neural tissues. Although the anti-HRP Ab does stain a small number of non-neural ectodermal cells in the grasshopper limb bud (Caudy and Bentley, 1986c), in number these cells represent less than $0.1 \%$ of the total number of non-neuronal cells that express either fasciclin I or fasciclin II during embryogenesis.

This paradox was resolved by the discovery that fasciclin I and II both come in heterogeneous glycosylated forms both with and without the neural-specific carbohydrate recognized by the anti-HRP Ab. This was shown by immunodepletion studies in which affinity-purified fasciclin I (Fig. $3 B$ ) and fasciclin II were subjected to multiple rounds of immunoprecipitation with excess anti-HRP Ab. After the first 3 rounds, no additional fasciclin I or II could be immunoprecipitated by anti-HRP, and yet after round 4 over $80 \%$ of the protein still remained and was immunoprecipitated by rat antisera against fasciclin I and
II, respectivcly. It is thus likcly that when fasciclin I and II are expressed on non-neural surfaces, they typically do not have the anti-HRP carbohydrate. However, it is not known whether all or only some of the fasciclin I and II molecules on neural surfaces have the carbohydrate. In summary, only about $10 \%$ of the fasciclin I molecules, as affinity-purified from grasshopper embryos, have the anti-HRP carbohydrate epitope.

\section{Conclusions}

In this paper, we have shown that antiserum against horseradish peroxidase (anti-HRP Ab), a widely used probe that labels the surfaces of neurons shortly after their birth in both Drosophila and grasshopper (Jan and Jan, 1982), recognizes a neural-specific carbohydrate moiety shared by many different surface glycoproteins in both grasshopper and Drosophila.

The discovery of a group of surface glycoproteins sharing a neural-specific carbohydrate epitope in Drosophila and grasshopper is reminiscent in certain respects of the $\mathrm{L} 2 / \mathrm{HNK}-1$ carbohydrate moiety expressed by many neural surface glycoproteins in vertebrates (Kruse et al., 1984, 1985). Many of the L2/ HNK-1 glycoproteins, including N-CAM, L1, MAG, and J1, appear to be involved in cell interactions and/or adhesion. By analogy, it is of interest to ask whether the anti-HRP glycoproteins might also be involved in cell interactions and/or adhesion during neuronal development in Drosophila and grasshopper. Quite unexpectedly, a different set of experiments (Bastiani et al., 1987) provided the proteins for an initial test of this hypothesis.

Previous studies on growth cone guidance in the CNS of the grasshopper embryo led to the proposal and experimental support of the "labeled pathways" hypothesis (Goodman et al., 1982; Raper et al., 1983a-c, 1984; Bastiani et al., 1984, 1986; Doe et al., 1986; du Lac et al., 1986), which predicts that axon fascicles in the embryonic neuropil are differentially labeled by surface recognition molecules used by growth cones for selective fasciculation. This model was further supported by recent experimental analysis of the fish embryo (Kuwada, 1986).

Monoclonal antibodies were used to identify potential candidates for such axonal recognition molecules. These studies led to the characterization and purification of 2 surface glycoproteins, fasciclin I and fasciclin II, which are expressed on different subsets of axon fascicles during development (Bastiani et al., 1987).

Here, we have shown that fasciclin I and II are anti-HRP glycoproteins. Thus, at least 2 of the anti-HRP glycoproteins are expressed on specific subsets of axon pathways during development and are good candidates for specific adhesion and/ or recognition molecules. Whether the other anti-HRP proteins will be of equal interest awaits future investigation.

Why do these 2 surface glycoproteins, with highly restricted neural expressions, share the same neural-specific carbohydrate epitope with each other and with many other neural glycoproteins? Given the possibility that glycoconjugates might serve as mediators or modulators of cell recognition and/or adhesion (Dodd et al., 1984), it would be of interest to use genetic analysis in Drosophila to alter the expression of this carbohydrate and thereby test its function.

Such experiments may now be possible, given the observation that Drosophila embryos homozygous for the TM3 chromosome lack this carbohydrate and yet express many, if not all, of the proteins. Whereas TM3 embryos lack the anti-HRP epitope from the outset of embryogenesis, a new mutation (which maps 
to the third chromosome) has recently been isolated (F. Katz and Y. N. Jan, personal communication) that abolishes the antiHRP epitope only after the beginning of pupal development. Thus, it should be possible to use genetic analysis to alter this neural-specific glycosylation and thus to test the function of this neural-specific carbohydrate.

\section{References}

Ashwell, G. (1966) New colorimetric methods of sugar analysis. Methods in Enzymol. 8: 85-95.

Bastiani, M. J., J. A. Raper, and C. S. Goodman (1984) Pathfinding by neuronal growth cones in grasshopper embryos. III. Selective affinity of the $\mathrm{G}$ growth cone for the P cells within the A/P fascicle. J. Neurosci. 4: 2311-2328.

Bastiani, M. J., S. du Lac, and C. S. Goodman (1986) Guidance of neuronal growth cones in the grasshopper embryo. I. Recognition of a specific axonal pathway by the pCC neuron. J. Neurosci. 6: 35183531 .

Bastiani, M. J., A. L. Harrelson, P. M. Snow, and C. S. Goodman (1987) Expression of fasciclin I and II glycoproteins on subsets of axon pathways during neuronal development in the grasshopper. Cell 48: 745755.

Bentley, D. H., and H. Keshishian (1982) Pathfinding by peripheral pioneer neurons in grasshoppers. Science 218: 1082-1088.

Blair, S. S., and J. Palka (1985a) Axon guidance in cultured wing discs and disc fragments of Drosophila. Dev. Biol. 108: 411-419.

Blair, S. S., and J. Palka (1985b) Axon guidance in the wing of Drosophila. Trends Neurosci. 8: 284-288.

Caudy, M., and D. Bentley (1986a) Pioneer growth cone morphologies reveal proximal increases in substrate affinity within leg segments of grasshopper embryos. J. Neurosci. 6: 364-379.

Caudy, M., and D. Bentley (1986b) Pioneering growth cones steering along a series of neuronal and non-neuronal cues of different affinities. J. Neurosci. 6: 1781-1795.

Caudy, M., and D. Bentley (1986c) Epithelial cell specialization at a limb segment boundary in the grasshopper embryo. Dev. Biol. 118 . 399-402.

Dodd, J., D. Solter, and T. M. Jessell (1984) Monoclonal antibodies against carbohydrate differentiation antigens identify subsets of primary sensory neurons. Nature 311: 469-472.

Doe, C. Q., M. J. Bastiani, and C. S. Goodman (1986) Guidance of neuronal growth cones in the grasshopper embryo. IV. Temporal delay experiments. J. Neurosci. 6: 3552-3563.

du Lac, S., M. J. Bastiani, and C. S. Goodman (1986) Guidance of neuronal growth cones in the grasshopper embryo. II. Recognition of a specific axonal pathway by the aCC neuron. J. Neurosci. $6: 3532-$ 3541 .

Edge, A. S. B., C. R. Faltynek, L. Hof, L. E. Reichert, and P. Weber (1981) Deglycosylation of glycoproteins by trifluoromethanesulfonic acid. Anal. Biochem. 118: 131-137.
Goodman, C. S., J. A. Raper, R. Ho, and S. Chang (1982) Pathfinding by neuronal growth cones in grasshopper embryos. Symp. Soc. Dev. Biol. 40: 275-316.

Goodman, C. S., M. J. Bastiani, C. Q. Doe, S. du Lac, S. L. Helfand, K. Y. Kuwada, and J. B. Thomas (1984) Cell recognition during neuronal development. Science 225: 1271-1279.

Greenwood, I. C., W. M. Hunter, and J. S. Glover (1963) The preparation of ${ }^{125}$ l labeled human growth hormone of high specific activity. J. Biochem. 84: 114-123.

Haustein, K., J. J. Marchalonis, and A. W. Harris (1975) Immunoglobulin of T lymphoma cells. Biosynthesis, surface representation and partial characterization. Biochemistry 14: 1826-1834.

Jan, L. Y., and Y. N. Jan (1982) Antibodies to horseradish peroxidase as specific neuronal markers in Drosophila and in grasshopper embryos. Proc. Natl. Acad. Sci. USA 79: 2700-2704.

Kruse, J., R. Mailhammer, H. Wernecke, A. Faissner, I. Sommer, C. Goridis, and M. Schachner (1984) Neural cell adhesion molecules and myelin-associated glycoprotein share a common carbohydrate moiety recognized by monoclonal antibodies L2 and HNK-1. Nature 311: 153-155.

Kruse, J., G. Keilhauer, A. Faissner, R. Timpl, and M. Schachner (1985) The J1 glycoprotein - a novel nervous system cell adhesion molecule of the L2/HNK-1 family. Nature 316: 146-148.

Kuwada, J. Y. (1986) Cell recognition by neuronal growth cones in a simple vertebrate embryo. Science 233: 740-746.

Laemmli, U. K. (1970) Cleavage of structural proteins during the assembly of the head of bacteriophage T4. Nature 227: 680-685.

Mitchison, T. J., and J. W. Sedat (1983) Localization of antigenic determinants in whole Drosophila embryos. Dev. Biol. 99: 261-264.

O'Farrell, P. (1975) High resolution two-dimensional electrophoresis of proteins. J. Biol. Chem. 250: 4007-4021.

Raper, J. A., M. J. Bastiani, and C. S. Goodman (1983a) Pathfinding by neuronal growth cones in grasshopper embryos: I. Divergent choices made by the growth cones of sibling neurons. J. Neurosci. 3: 20-30.

Raper, J. A., M. J. Bastiani, and C. S. Goodman (1983b) Pathfinding by neuronal growth cones in grasshopper embryos: II. Selective fasciculation onto specific axonal pathways. J. Neurosci. 3: 31-41.

Raper, J. A., M. J. Bastiani, and C. S. Goodman (1983c) Guidance of neuronal growth cones: Selective fasciculation in the grasshopper embryo. Cold Spring Harbor Symp. Quant. Biol. 48: 587-598.

Raper, J. A., M. J. Bastiani, and C. S. Goodman (1984) Pathfinding by neuronal growth cones in grasshopper embryos: IV. The effects of ablating the $A$ and $P$ axons upon the behavior of the $G$ growth cone. J. Neurosci. 4: 2329-2345.

Spiro, R. G. (1966) Analysis of sugars found in glycoproteins. Methods Enzymol. 8: 26-52.

van Agthoven, A., C. Terhorst, E. Reinherz, and S. Schlossman (1981) Characterization of $T$ cell surface glycoproteins $T 1$ and $T 3$ present on all human peripheral $\mathrm{T}$ lymphocytes and functionally mature thymocytes. Eur. J. Immunol. 11: 18-21.

Wiedenmann, B., and W. W. Franke (1985) Identification and localization of synaptophysin, an integral membrane glycoprotein of $M_{\mathrm{r}}$ 38,000 characteristic of presynaptic vesicles. Cell 41: 1017-1028. 